\title{
Potencial do Uso de Amostras Fecais de Felinos como Ferramenta de Monitoramento Ambiental: Estudo de Caso no Parque Nacional da Serra dos Órgãos
}

\author{
Cecilia Cronemberger ${ }^{1,5}$, Rafael Conceição de Moura², Marina Lopes Duarte ${ }^{3}$, Lais Verdan Dib ${ }^{3}$, Alynne da Silva Barbosa ${ }^{3,6}$, \\ Leticia Oliveira ${ }^{4}$, Roberta Loh ${ }^{4}$, Maria Regina Reis Amendoeira ${ }^{3} \&$ Helena Godoy Bergallo ${ }^{5}$
}

Recebido em 14/09/2020 - Aceito em 15/09/2021

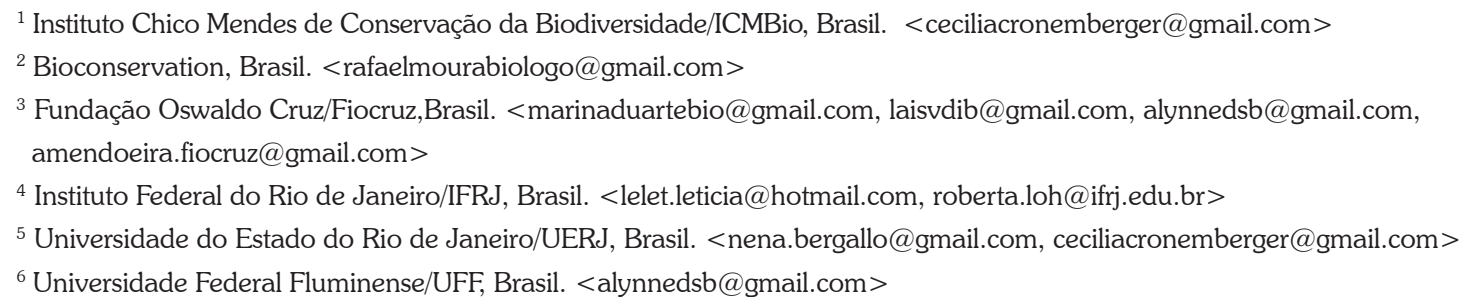

RESUMO - O monitoramento das unidades de conservação (UCs) é fundamental para avaliar se atingem seus objetivos. Felinos são bons indicadores ambientais e suas fezes podem fornecer informações relevantes para o monitoramento de UCs. Utilizamos fezes encontradas em trilhas do Parque Nacional da Serra dos Órgãos para registrar a presença de felinos e outros mamíferos, analisamos sua distribuição espacial e discutimos o potencial do uso dessas amostras como ferramenta de monitoramento ambiental. Entre 2009 e 2019, foram coletadas 486 amostras em 21 trilhas. Foi possível identificar um mamífero em $61 \%$ das 315 amostras analisadas por microscopia óptica dos pelos-guarda. As cinco espécies de felinos com ocorrência confirmada no parque foram registradas, com destaque para o gato maracajá (Leopardus wiedii), frequentemente registrado em altitudes mais elevadas que o conhecido. Foram identificados mamíferos com poucos registros anteriores no parque, como o mão-pelada (Procyon cancrivorous). A distribuição espacial das amostras reflete a altimetria da área amostrada, com uma concentração nos campos de altitude, onde outros métodos têm-se mostrado menos eficazes no registro de mamíferos, destacando-se a importância de usar métodos complementares de monitoramento. Fezes podem ser utilizadas em análises moleculares, que, além de refinar a identificação taxonômica, podem desvendar informações como o grau de isolamento genético das populações. As fezes podem fornecer informações sobre a saúde animal através do estudo de parasitos gastrointestinais, que também são bons indicadores de qualidade ambiental e sobre contaminação ambiental. Apesar de seu potencial, o uso de fezes de felinos como ferramenta de monitoramento ainda é raro.

Palavras-chave: Tricologia; fezes; Felidae; monitoramento; unidade de conservação.

\section{Potential of the use of Feline Fecal Samples as an Environmental Monitoring Tool: Case Study in Serra dos Órgãos National Park}

ABSTRACT - Monitoring of Protected Areas (PAs) is essential to assess whether they achieve their goals. Felines are good environmental indicators, and their feces can provide relevant information for PA monitoring. We used feces found on the trails of the Serra dos Órgãos National Park to record the presence of felines and other mammals. We analyzed their spatial distribution and discussed the potential of using these samples as an environmental monitoring tool. Between 2009 and 2019, 486 samples were collected on 21 trails. It was possible to identify mammal species in $61 \%$ of the 315 samples analyzed by guard hair optical microscopy. The five feline species with confirmed occurrence in the park were registered, with emphasis on the margay (Leopardus wiedii), frequently registered in higher altitudes than previously known. Mammals with few records in the park were identified, such as crab-eating raccoon (Procyon cancrivorous). The spatial distribution of the samples reflects the altimetry of the sampled area, with a concentration in the "campos de altitude", where other methods are less effective in registering mammals, highlighting the importance of using complementary monitoring methods. Feces can be used in molecular analysis, which, in addition to refining taxonomic identification, can reveal information such as the degree of genetic isolation of populations. They can provide information on animal health through the study of gastrointestinal parasites, which are also good indicators of environmental quality, and on environmental contamination. Despite its potential, the use of feline feces as a monitoring tool is still rare.

Keywords: Trichology; feces; Felidae; monitoring; protected area. 


\section{Potencial del Uso de Muestras Fecales de Felinos como Herramienta de Monitoreo Ambiental: Estudio de Caso en el Parque Nacional Serra dos Órgãos}

RESUMEN - El monitoreo de las Areas Protegidas (AP) es esencial para evaluar si logran sus objetivos. Los felinos son buenos indicadores ambientales y sus heces pueden proporcionar información relevante para monitorear las AP. Usamos heces encontradas en senderos en el Parque Nacional Serra dos Órgãos para registrar la presencia de felinos y otros mamíferos, analizar su distribución espacial y discutir el potencial de usar estas muestras como una herramienta de monitoreo ambiental. Entre 2009 y 2019 , se recolectaron 486 muestras en 21 senderos. Fue posible identificar un mamífero en $61 \%$ de las 315 muestras analizadas por microscopía óptica de pelos de guardia. Se registraron las cinco especies de felinos con presencia confirmada en el parque, con énfasis en el margay (Leopardus wiedii), frecuentemente registrado en altitudes superiores a las conocidas anteriormente. Se han identificado mamíferos con pocos registros previos en el parque, como el mapache cangrejero (Procyon cancrívoro). La distribución espacial de las muestras refleja la altimetría del área muestreada, con una concentración en los "campos de altitud", donde otros métodos han demostrado ser menos efectivos en el registro de mamíferos, destacando la importancia de utilizar métodos de monitoreo complementarios. Las heces se pueden utilizar en análisis moleculares que, además de refinar la identificación taxonómica, pueden revelar información como el grado de aislamiento genético de las poblaciones. Las heces pueden proporcionar información sobre la salud animal mediante el estudio de los parásitos gastrointestinales, que también son buenos indicadores de la calidad ambiental, y sobre la contaminación ambiental. A pesar de su potencial, el uso de heces felinas como herramienta de monitoreo sigue siendo poco común.

Palabras-clave: Tricología; heces; Felidae; monitoreo; área protegida.

\section{Introdução}

A criação de áreas protegidas tem sido uma das principais estratégias de conservação da natureza adotadas ao redor do mundo (McDonald \& Boucher, 2011). No entanto, o simples estabelecimento de tais áreas não garante a efetiva conservação de sua biodiversidade, sendo necessário garantir sua gestão efetiva (Bruner et al., 2001). Nesse sentido, o monitoramento da biodiversidade é uma ferramenta importante para acompanhar a qualidade dos ecossistemas e suas respostas a mudanças ambientais e ações de manejo (Lindenmayer \& Likens, 2009; Lovett et al., 2007), e assim, avaliar se as áreas protegidas estão atendendo seus objetivos de criação.

Os mamíferos silvestres provêm importantes serviços ecossistêmicos, como polinização, dispersão de sementes e controle de pragas (Ahumada et al., 2011). Mamíferos de médio e grande porte desempenham importante papel ecológico nos ecossistemas, seja por meio de controle populacional das espécies de presa, no caso de carnívoros, seja na engenharia da estrutura e composição do ambiente, no caso de herbívoros (Morrison et al., 2011) e insetívoros (Fontes et al., 2020).
Suas características naturais, como longo período de gestação, baixa densidade populacional $e$ necessidade de grandes áreas, aliadas à pressão de caça, tornam os mamíferos de médio e grande porte um dos grupos mais suscetíveis à extinção (Cullen Jr et al., 2005; Galleti et al., 2013, Rodríguez-Castro et al., 2020).

Felídeos e canídeos, em particular, têm sido utilizados como indicadores de qualidade ambiental, já que suas necessidades ecológicas podem abranger as áreas de vida de várias outras espécies (Jorge et al., 2013). No entanto, devido aos seus hábitos crípticos e densidade naturalmente baixas, esses animais são difíceis de observar e estudar (Rodríguez-Castro et al., 2020; Elbroch et al., 2015).

Recentemente, novos métodos não invasivos para o estudo desses animais têm surgido, como o uso das armadilhas fotográficas, mas que ainda assim apresentam um custo considerável, seja do equipamento ou da logística de manutenção em campo (Abrams et al., 2018; Wearn \& GloverKapfer, 2017). Métodos como análise da morfologia e conteúdo fecal e a identificação microscópica de pelos (tricologia) constituem alternativas não invasivas e de baixo custo (Quadros \& MonteiroFilho, 2006a), que permitem a coleta oportunística de amostras e, portanto, aumentam a eficiência do trabalho de inventário e monitoramento. 
Os felinos têm o hábito de autolimpeza e acabam ingerindo e defecando seus próprios pelos (Chame, 2003), o que permite usar os pelosguarda encontrados nas fezes para identificação taxonômica. Os pelos-guarda geralmente são pelos mais longos e sobressalentes na pelagem dos animais, sendo utilizados na identificação de espécies de mamíferos, já que a combinação de características de sua cutícula, medula e córtex confere informações diagnósticas específicas a uma determinada espécie (Quadros \& MonteiroFilho, 2006b).

Além disso, por serem animais carnívoros, nas fezes de felinos também são encontrados pelos dos animais que consumiram e outros restos, como ossos, garras e penas. Assim, amostras fecais de felinos permitem identificar, por tricologia $e$ outras técnicas, não apenas a quem pertencem as fezes, mas uma ampla gama de animais que fazem parte da sua dieta, como mamíferos, aves, répteis, entre outros (Wang, 2002), podendo ser usadas como ferramenta complementar de inventário de comunidades.

Este artigo apresenta os resultados da identificação de mamíferos por meio da tricologia de pelos encontrados em amostras fecais de felinos coletadas no Parque Nacional da Serra dos Órgãos e sua distribuição espacial. Adicionalmente, discutimos sobre as possibilidades de uso desse método como ferramenta de monitoramento ambiental em unidades de conservação.

\section{Material e Métodos \\ Área de estudo}

O Parque Nacional da Serra dos Órgãos (PARNASO) está localizado na cadeia de montanhas da Serra do Mar no estado do Rio de Janeiro, Brasil, e apresenta uma área de 20.024 hectares, abrangendo os municípios de Teresópolis, Petrópolis, Magé e Guapimirim (Cronemberger \& Viveiros de Castro, 2007). O PARNASO é parte do Mosaico de Unidades de Conservação da Mata Atlântica Central Fluminense, que abriga uma grande extensão contínua de floresta montana e alto-montana com altos índices de diversidade e endemismo, sendo um refúgio importante para espécies ameaçadas de mamíferos, anfíbios e répteis da Mata Atlântica (Galindo-Leal et al., 2005).
O relevo acidentado, com altitudes variando entre cerca de 200 a $2300 \mathrm{~m}$ acima do nível do mar, é uma das características mais marcantes do PARNASO. O clima do PARNASO é mesotérmico e superúmido, caracterizado por temperaturas amenas (média anual entre $13 \mathrm{e}$ $23^{\circ} \mathrm{C}$ ) e chuva abundante, influenciada pelo efeito orográfico, o que faz da pluviosidade local a mais elevada do estado, chegando a $3000 \mathrm{~mm}$ anuais (Cronemberger \& Viveiros de Castro, 2007). Em resposta a essa grande variação altitudinal, são reconhecidos na área do parque quatro formações vegetacionais: floresta pluvial submontana; floresta pluvial montana; floresta pluvial alto-montana, também conhecida como mata nebular, e, por fim, os campos de altitude. Os campos estão nos topos das montanhas, a partir de cerca de $1800 \mathrm{~m}$ de altitude, onde a floresta é substituída por uma formação aberta, dominada por plantas herbáceo-arbustivas (Cronemberger \& Viveiros de Castro, 2007).

A fauna também é influenciada pela diversidade de habitat criada pela variação na topografia e na flora. Com relação a mamíferos, há registros recentes da ocorrência de 200 espécies, sendo 26 classificadas em alguma categoria de ameaça (Cronemberger et al., 2019), além de quatro espécies consideradas localmente extintas: onça pintada, Panthera onca (Linnaeus, 1758), anta, Tapirus terrestris (Linnaeus, 1758), queixada, Tayassu pecari (Link, 1795) e oveado (Mazama sp.). Cinco espécies de felinos silvestres têm ocorrência recente confirmada no PARNASO: onça parda ou suçuarana, Puma concolor (Linnaeus, 1771), jaguatirica, Leopardus pardalis (Linnaeus, 1758), gato maracajá, Leopardus wiedii (Schinz, 1821), gato-do-mato-pequeno, Leopardus guttulus (Hensel, 1872) e o gato mourisco, Herpailurus yagouaroundi (É. Geoffroy, 1803).

\section{Amostragem}

Fezes de mamíferos, principalmente aquelas com morfologia similar à de felídeo, segundo o padrão proposto por Chame (2003), vêm sendo coletadas sempre que são encontradas nas trilhas pela equipe de funcionários e colaboradores do PARNASO, desde setembro de 2009. Entre 2009 e 2019, foram coletadas 486 amostras fecais (Tabela 1). 
Tabela 1 - Número de amostras fecais coletadas por ano. SI $=$ sem informação.

\begin{tabular}{|c|c|}
\hline Ano & Número de amostras \\
\hline 2009 & 2 \\
\hline 2010 & 19 \\
\hline 2011 & 63 \\
\hline 2012 & 87 \\
\hline 2013 & 121 \\
\hline 2014 & 60 \\
\hline 2015 & 70 \\
\hline 2016 & 22 \\
\hline 2017 & 3 \\
\hline 2018 & 0 \\
\hline 2019 & 32 \\
\hline SI & 7 \\
\hline Total & 486 \\
\hline
\end{tabular}

As amostras foram coletadas seguindo o protocolo proposto por Moura \& Faria (2012a), que consiste em utilizar saco plástico limpo para recolher e armazenar uma parte da amostra fecal, deixando sempre um fragmento de cerca de 4 $\mathrm{cm}$ no local, mantendo a marcação territorial dos mamíferos. Apenas uma amostra foi armazenada por saco plástico, registrando a descrição precisa do local e/ou coordenadas geográficas na ficha de campo e no saco plástico (Fig. 1).

\section{É comum encontrar várias amostras} na mesma latrina, em laje de pedra, especialmente nos campos de altitude (Fig. 1B). Nesse caso, asfezesforamarmazenadaseregistradas individualmente. Logo após a coleta, as amostras foram armazenadas em freezer no laboratório do Centro de Referência em Biodiversidade da Serra dos Órgãos, PARNASO, até o momento da análise.
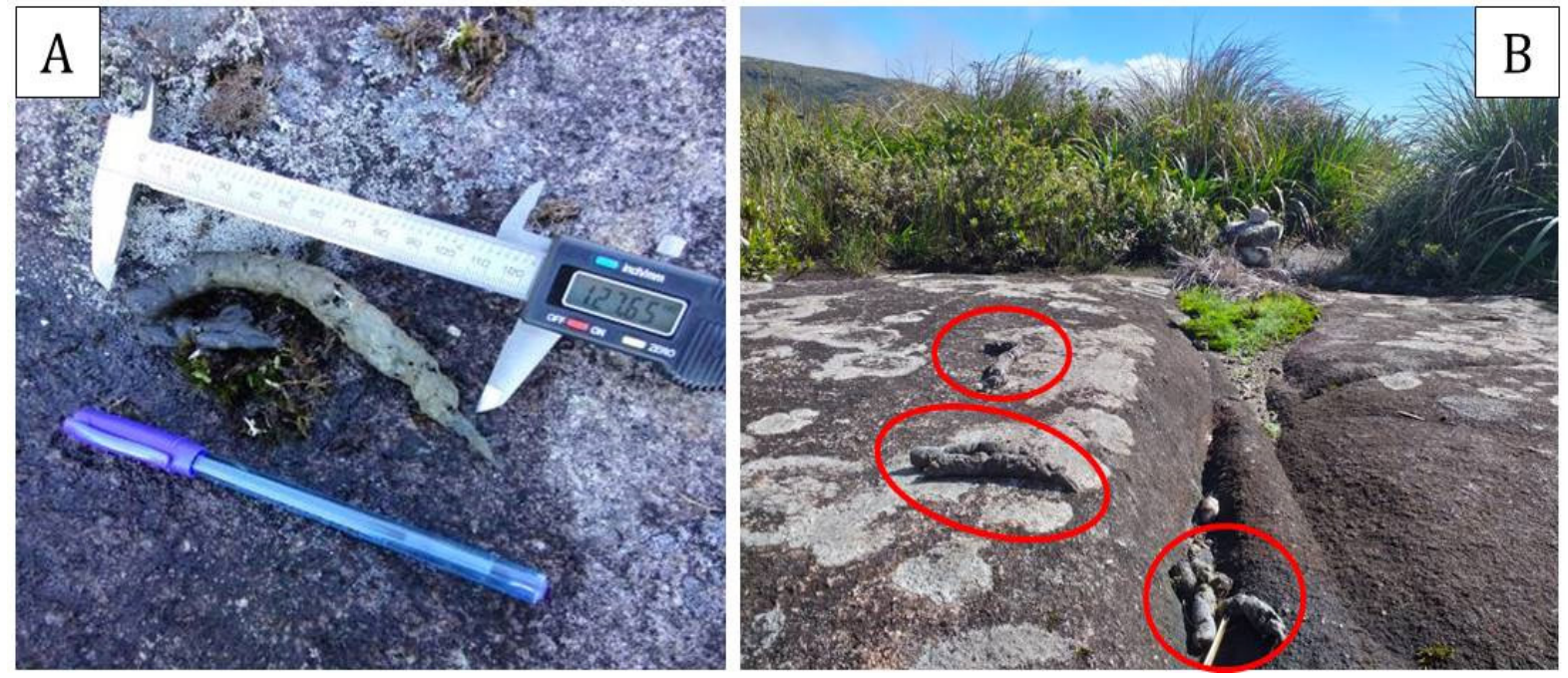

Figura 1 - Coleta de amostras fecais em campo (A). Latrina em laje de pedra, com mais de uma amostra.

A maior parte das amostras foi coletada de forma oportunística, isto é, ao percorrer as trilhas com outros objetivos, como fiscalização, manutenção de trilhas ou outras pesquisas. Dessa forma, não há registro do esforço total empregado, ou seja, da quilometragem total percorrida por todas as pessoas que coletaram amostras ao longo dos anos, mas apenas o registro dos dias em que houve coleta de amostras.

\section{Processamento laboratorial das amostras}

A identificação taxonômica dos mamíferos foi feita por meio da análise dos pelos-guarda baseada no protocolo estabelecido por Quadros \& MonteiroFilho (2006a). Para recuperação dos pelos-guarda, as fezes coletadas foram lavadas com água corrente sobre uma peneira de malha fina com gramatura de $1 \mathrm{~mm}$. Após a secagem em estufa, os pelos recuperados foram triados, sendo os pelos-guarda encontrados separados de acordo com comprimento e similaridades morfológicas e posteriormente armazenados em sacos plásticos, devidamente identificados. Além disso, restos de remanescentes alimentares tais 
como ossos, dentes, unhas, garras, escamas, bicos, penas foram separados e armazenados em sacos plásticos específicos. A identificação taxonômica de mamíferos ocorreu por meio de duas técnicas tricológicas: a impressão cuticular e a visualização medular dos pelos-guarda presentes nas fezes. A técnica de impressão cuticular consiste em prensar, com ajuda de morsa, o pelo-guarda em uma lâmina de vidro com uma camada de esmalte incolor. Depois de 30 minutos, o pelo é retirado e o resultado é uma lâmina que permite observar, ao microscópio óptico, o padrão das escamas cuticulares do pelo (Fig. $3 \mathrm{~A}$, C, E e G). Em seguida, o mesmo pelo foi diafanizado com água oxigenada e fixado em nova lâmina de microscopia com uma gota de Entellan ${ }^{\circledR}$ e lamínula (Fig. $3 \mathrm{~B}, \mathrm{D}, \mathrm{F}$ e H), seguindo o protocolo proposto por Quadros \& Monteiro-Filho (2006a).

A leitura das lâminas com as impressões cuticulares e medulares foi realizada em microscópio óptico binocular em aumento de 100 e 400 vezes.
A identificação dos pelos guarda foi feita com o auxilio de literatura pertinente (Quadros, 2002; Miranda et al., 2014), além da utilização da coleção de referência de pelos de mamíferos do PARNASO (Moura \& Faria, 2012b).

\section{Análise espacial}

As 486 amostras foram coletadas em 21 trilhas, organizadas em 15 regiões dentro do PARNASO (Fig. 2, Tabela 2). Para ilustrar a distribuição espacial das amostras e das espécies de mamíferos identificadas, elaboramos mapas utilizando o software ArcGIS Pro 2.6.3 (ESRI, 2020) sobre mapa base desenvolvido por Earthstar Geographics, usando as coordenadas geográficas anotadas em campo. Foi possível mapear 468 das 486 amostras.

Tabela 2 - Extensão das trilhas em que foram coletadas amostras, número de amostras e número de ocasiões diferentes em que foram coletadas amostras em cada uma. A informação sobre região se refere às legendas das Figuras 2 e 5 . NA = não se aplica.

\begin{tabular}{|c|c|c|c|c|}
\hline Região & Trilha & Extensão (m) & Número de amostras & Número de ocasiões \\
\hline 1 & Rio Soberbo & 1404 & 3 & 2 \\
\hline 2 & Trilha do Dedo de Nossa Senhora & 1018 & 2 & 1 \\
\hline 2 & Trilha do Dedo de Deus & 846 & 1 & 1 \\
\hline 2 & Trilha para Cabeça de Peixe & 1057 & 2 & 2 \\
\hline 3 & Trilha 360 & 2541 & 1 & 1 \\
\hline 3 & Trilha Mozart Catão & 934 & 1 & 1 \\
\hline 3 & Trilha Suspensa & 989 & 4 & 3 \\
\hline 4 & Trilha do Rancho Frio & 2608 & 10 & 7 \\
\hline 5 & Caminho das Orquídeas & 2040 & 6 & 1 \\
\hline 5 & Trilha da Pedra do Sino & 8956 & 218 & 36 \\
\hline 6 & Trilha Açu-Sino & 7455 & 133 & 18 \\
\hline 7 & Morro do Alicate & 774 & 5 & 1 \\
\hline 7 & Trilha para Açu & 7008 & 55 & 20 \\
\hline 8 & Trilha para Cubaio & 3306 & 7 & 1 \\
\hline 9 & Vale do Jacó & 861 & 1 & 1 \\
\hline 10 & Fazenda Boa Esperanca & 731 & 6 & 1 \\
\hline 11 & Trilha Uricanal & 3622 & 2 & 2 \\
\hline 12 & Trilha Caxambú-Açu & 7561 & 6 & 3 \\
\hline 13 & Caxambu Grande & 3536 & 6 & 4 \\
\hline 14 & Travessia Cobiçado-Ventania & 9101 & 10 & 3 \\
\hline \multirow[t]{3}{*}{15} & Morin & 505 & 1 & 1 \\
\hline & Sem Informação & NA & 6 & NA \\
\hline & Total & \multicolumn{3}{|c|}{486} \\
\hline
\end{tabular}




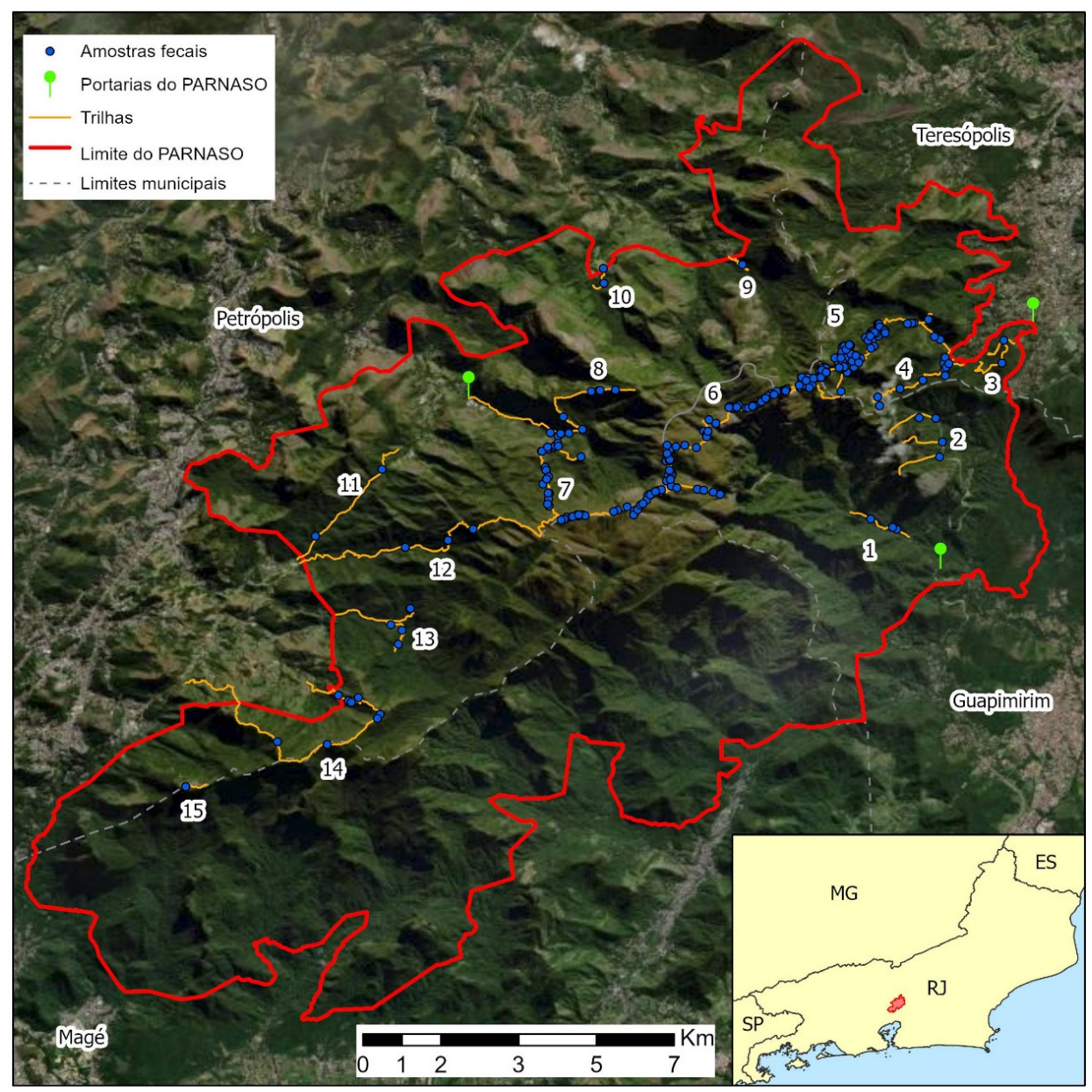

Figura 2 - Localização de 468 amostras fecais com coordenadas geográficas coletadas no Parque Nacional da Serra dos Órgãos, estado do Rio de Janeiro. As 21 trilhas estão agrupadas em 15 regiões, indicadas na Tabela 2. Mapa base: Earthstar Geographics.

Usamos um Modelo Digital de Terreno ALOS PALSAR RTC (AFC DAAC 2014) e a extensão Spatial Analyst do software ArcGis Pro (ESRI 2020) para determinar a altitude das amostras e o perfil altimétrico das trilhas. Consideramos a área amostral de cada trilha como sendo uma área de $1,5 \mathrm{~m}$ de cada lado do curso das trilhas e calculamos a proporção da área de cada trilha em cada faixa de $100 \mathrm{~m}$ de altitude, bem como a proporção da área amostral total em cada altitude.

Para possibilitar a comparação entre trilhas com esforço amostral desconhecido, utilizamos o número de amostras encontradas por ocasião (amostras/ocasião), calculado pela divisão do número de amostras encontradas em cada faixa altitudinal de cada trilha pelo número de ocasiões em que houve coleta de amostras naquela trilha.
Para avaliar se a distribuição altitudinal das amostras é consequência unicamente da área amostrada em cada faixa altitudinal, realizamos um teste de Qui-quadrado, utilizando o software RStudio 1.3.1093 (RStudio Team 2020), considerando duas hipóteses alternativas: $\mathrm{H} 0$ ) a probabilidade de encontrar fezes de felinos é a mesma em qualquer altitude, e, portanto, a distribuição das frequências de amostras encontradas por ocasião em cada faixa altitudinal não é significativamente diferente da proporção de área amostral em cada faixa e H1) A probabilidade de encontrar fezes de felinos não é a mesma em qualquer altitude. Consideramos o percentual da área amostral total em cada faixa de $100 \mathrm{~m}$ como a proporção esperada de amostras, dado o perfil altimétrico das trilhas percorridas, e a proporção de amostras/ocasião por faixa altitudinal como a proporção encontrada. Consideramos na análise apenas as trilhas onde houve mais de 10 ocasiões de coleta. 


\section{Resultados}

Das 486 amostras coletadas, 315, coletadas até 2015 , foram processadas usando os métodos da tricologia. Foi possível classificar taxonomicamente os pelos-guarda recuperados como pertencentes a mamíferos em 191 amostras (61\% do total), sendo duas em nível de ordem, quatro em nível de família, duas em nível de gênero e 183 em nível de espécie (Tabela 3 e Fig. 3). Em 79\% dos casos, o pelo analisado foi identificado como sendo de felino, e em $21 \%$ dos casos, de outros mamíferos.

Tabela 3 - Mamíferos identificados pela tricologia dos pelos-guarda recuperados nas amostras fecais e respectivas características diagnósticas da cutícula e medula dos pelos. NI - Não Identificado.

\begin{tabular}{|c|c|c|c|}
\hline $\begin{array}{c}\text { Mamíferos } \\
\text { identificados }\end{array}$ & $\begin{array}{l}\text { Quantidade de } \\
\text { amostras (\%) }\end{array}$ & Cutícula & Medula \\
\hline Leopardus wiedii & $70(37 \%)$ & $\begin{array}{l}\text { Escamas imbricadas com formato } \\
\text { folidáceo muito estreita }\end{array}$ & $\begin{array}{c}\text { Multisseriada, larga com } \\
\text { Margens fimbriadas e células trabeculares }\end{array}$ \\
\hline Leopardus pardalis & $30(16 \%)$ & $\begin{array}{l}\text { Escamas imbricadas } \\
\text { com formato folidáceo } \\
\text { intermediário }\end{array}$ & $\begin{array}{c}\text { Multisseriada, larga com } \\
\text { Margens fimbriadas e células trabeculares }\end{array}$ \\
\hline Leopardus guttulus & $29(15 \%)$ & $\begin{array}{c}\text { Escamas pavimentosas, losângicas } \\
\text { e estreitas }\end{array}$ & $\begin{array}{c}\text { Multisseriada, larga com } \\
\text { Margens fimbriadas e células trabeculares }\end{array}$ \\
\hline H. yagouaroundi & $16(8 \%)$ & $\begin{array}{c}\text { Escamas pavimentosas organizadas } \\
\text { em mosaico ou losângica larga e } \\
\text { bordas levemente ornamentadas }\end{array}$ & $\begin{array}{l}\text { Multisseriada, larga com } \\
\text { Margens fimbriadas e células trabeculares }\end{array}$ \\
\hline Puma concolor & $2(1 \%)$ & $\begin{array}{l}\text { Escamas pavimentosa ondeada, } \\
\text { larga, transversal, lisa e contínua }\end{array}$ & $\begin{array}{c}\text { Multisseriada, larga com } \\
\text { Margens fimbriadas e células trabeculares }\end{array}$ \\
\hline Família Felidae & $4(2 \%)$ & $\mathrm{NI}$ & $\begin{array}{c}\text { Multisseriada, larga com } \\
\text { Margens fimbriadas e células trabeculares }\end{array}$ \\
\hline Cerdocyon thous & $1(1 \%)$ & $\begin{array}{l}\text { Escamas ondeadas e transversais } \\
\text { Bordas ornamentadas }\end{array}$ & $\begin{array}{c}\text { Multisseriada, larga, margens íntegras e } \\
\text { células anisocélicas }\end{array}$ \\
\hline Didelphis aurita & $5(3 \%)$ & $\begin{array}{l}\text { Ondeada, irregular, com bordas } \\
\text { das } \\
\text { escamas incompletas }\end{array}$ & Simples e crivada \\
\hline Eira barbara & $4(2 \%)$ & $\begin{array}{c}\text { Escamas pavimentosa, ondeada, } \\
\text { transversal e com borda lisa e } \\
\text { contínua }\end{array}$ & $\begin{array}{l}\text { Multisseriada, larga, margem crenada } \\
\text { células trabeculares }\end{array}$ \\
\hline Gracilianus microtarsus & $2(5 \%)$ & Conoidal simétrica & Continua, multisseriada e escalariforme \\
\hline Lontra longicaudis & $3(8 \%)$ & $\begin{array}{c}\text { Escamas imbricada, folidáce e } \\
\text { estreita }\end{array}$ & $\begin{array}{l}\text { Contínua, intermediária, multiseriada, } \\
\text { anastomosada, trabecular e crenada }\end{array}$ \\
\hline Nasua nasua & $9(23 \%)$ & $\begin{array}{c}\text { Escamas pavimentosas, ondeadas, } \\
\text { irregulares com margens } \\
\text { ornamentadas }\end{array}$ & $\begin{array}{l}\text { Multisseriada, larga, margem íntegra, } \\
\text { células trabeculares }\end{array}$ \\
\hline Procyon cancrivorus & $5(13 \%)$ & $\begin{array}{c}\text { Escamas pavimentosas, ondeadas, } \\
\text { irregulares com margens } \\
\text { ornamentadas }\end{array}$ & $\begin{array}{l}\text { Multisseriada, estreita, margem íntegra, } \\
\text { células anastomosadas com formato amorfo }\end{array}$ \\
\hline Pecari tajacu & $1(3 \%)$ & $\begin{array}{l}\text { Pavimentosa, ondeada e transver- } \\
\text { sal }\end{array}$ & $\begin{array}{l}\text { Multisseriada e celulas isoladas com } \\
\text { formato cordonal }\end{array}$ \\
\hline Ordem Rodentia & $2(5 \%)$ & $\mathrm{NI}$ & Multiseriada alveolar \\
\hline Total & 191 & & \\
\hline
\end{tabular}


Figura 3 - Cutícula (A, C, E e G) e medula (B, D, F e H) de pelos-guarda de espécies de mamíferos identificados no Parnaso no período de 2009 a 2019. A e B. Leopardus wiedii. Ce D. Cerdocyon thous. E e F. Lontra longicaudis. G e H. Roedor.
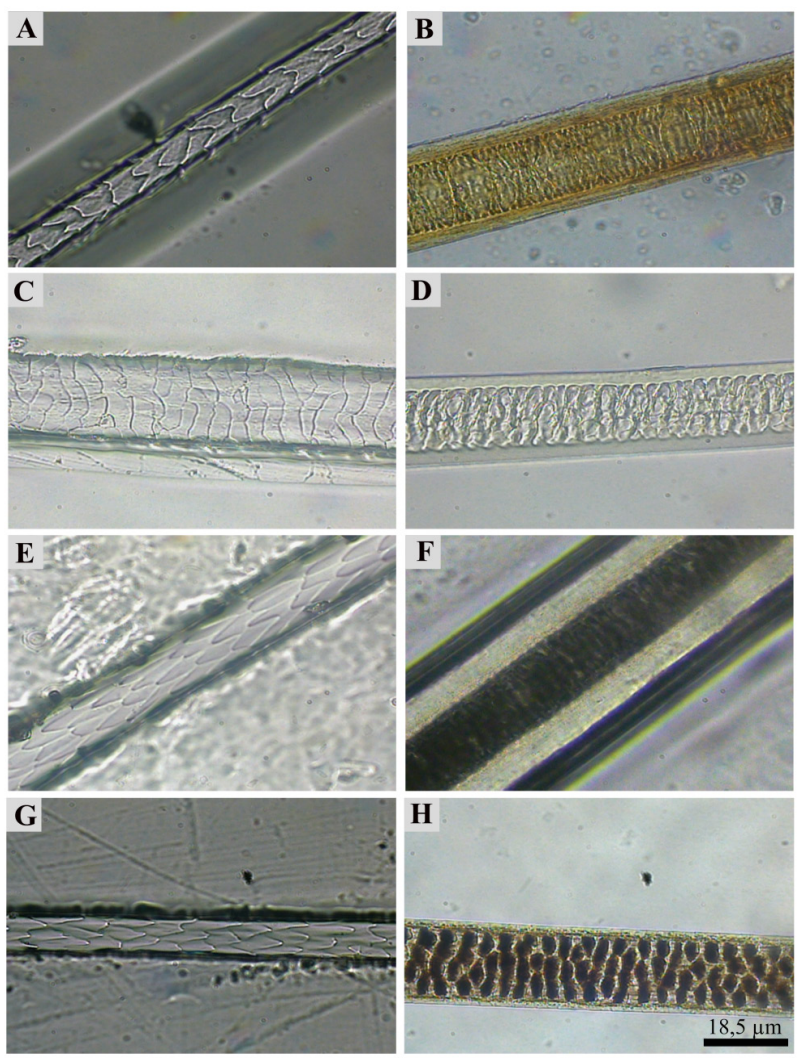

Na maioria das amostras analisadas, foram recuperados pelos-guarda de felinos de porte médio, destacando-se o gato-maracajá (Leopardus wiedii), seguido de jaguatirica (Leopardus pardalis), gato-domato-pequeno (Leopardus guttulus) e gato mourisco (Puma yagouaroundi). Apenas duas amostras foram atribuídas à onça parda (Puma concolor), o maior felino registrado no PARNASO,

Outras espécies de carnívoros também foram identificadas nas amostras, incluindo cachorro-do-mato (Cerdocyon thous, Linnaeus 1766), irara (Eira barbara, Linnaeus 1758), furão pequeno (Galictis cuja, Molina 1782), lontra (Lontra longicaudis, Olfers 1818), quati (Nasua nasua, Linnaeus 1766) e mão pelada (Procyon cancrivorus, G. Cuvier 1798). Além disso, pelosguarda de mamíferos de outros táxons também foram identificados no material fecal, incluindo pelos de roedores e marsupiais, como Gracilinanus microtarsus (Wagner 1842), e o cateto (Pecari tajacu, Linnaeus 1758) (Tabela 3).

As amostras fecais foram coletadas principalmente nos campos de altitude, formação aberta que ocorre acima de $1800 \mathrm{~m}$ do nível do mar, correspondendo a $72 \%$ das amostras. A faixa altitudinal que concentrou a maior quantidade de amostras foi entre 2001 a 2100m acima do nível do mar (Fig. 3). As amostras atribuídas a felinos também se concentraram nas altitudes mais elevadas, com exceção de Puma concolor (Fig. 3).

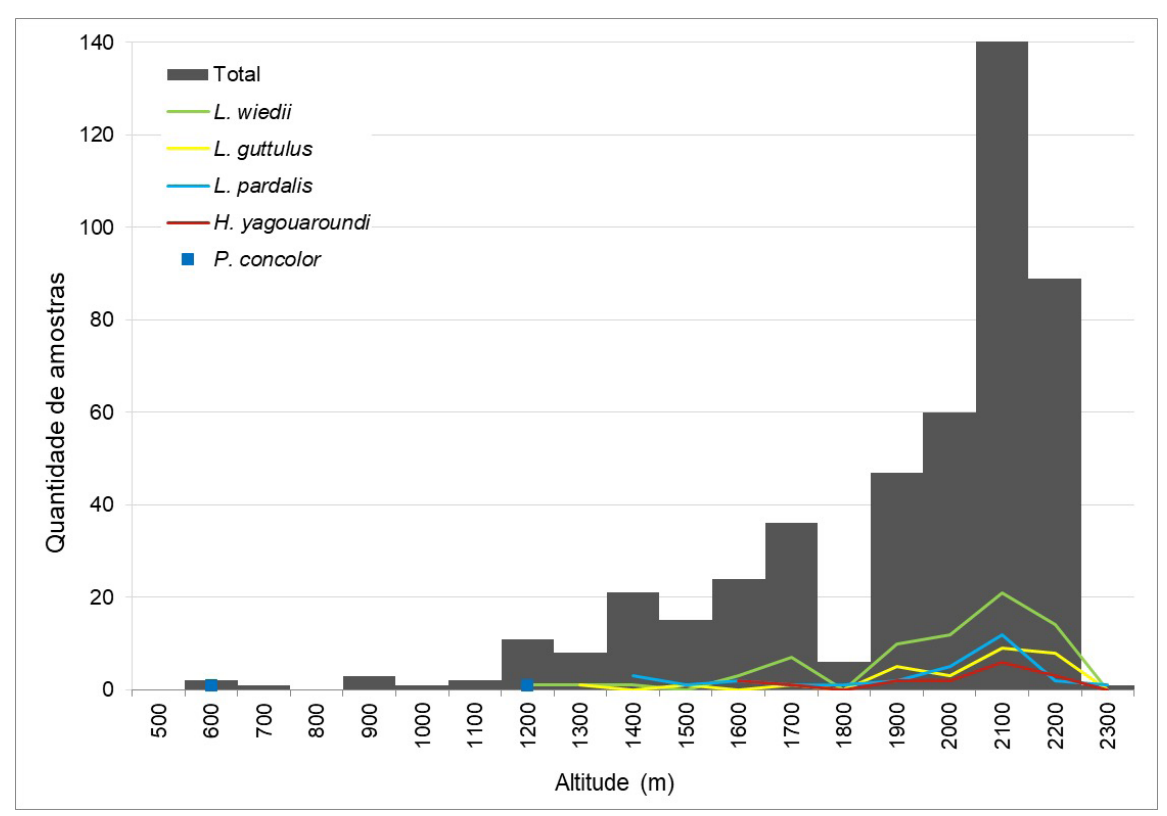

Figura 3 - Quantidade de amostras coletadas por altitude, com destaque para as amostras atribuídas a felinos. 
As trilhas com maior número de amostras coletadas foram a Trilha da Pedra do Sino, com 212 amostras, seguida pela Trilha Açu-Sino, com 126 amostras, e pela Trilha do Açu, com 58 amostras. Essas trilhas, juntas, representam a Travessia Petrópolis-Teresópolis, o principal eixo de deslocamento pela porção central do PARNASO, onde se concentram as maiores altitudes $e$ a fitofisionomia dos campos de altitude (Fig. 4); estão entre as mais longas das trilhas amostradas e foram amostradas mais vezes, sendo as únicas trilhas com mais de 10 ocasiões amostrais (Tabela 2). Essas trilhas concentraram a maior parte das amostras atribuídas aos felinos de médio porte (Leopardus wiedii, L. gutullus, L. pardalis e $H$. yagouarondi), mas nenhuma das duas amostras atribuídas a $P$. concolor (Fig. 4), coletadas nas trilhas do rio Soberbo e Caxambu Grande. O uso dessa área pelos felinos de médio porte foi recorrente ao longo dos anos.

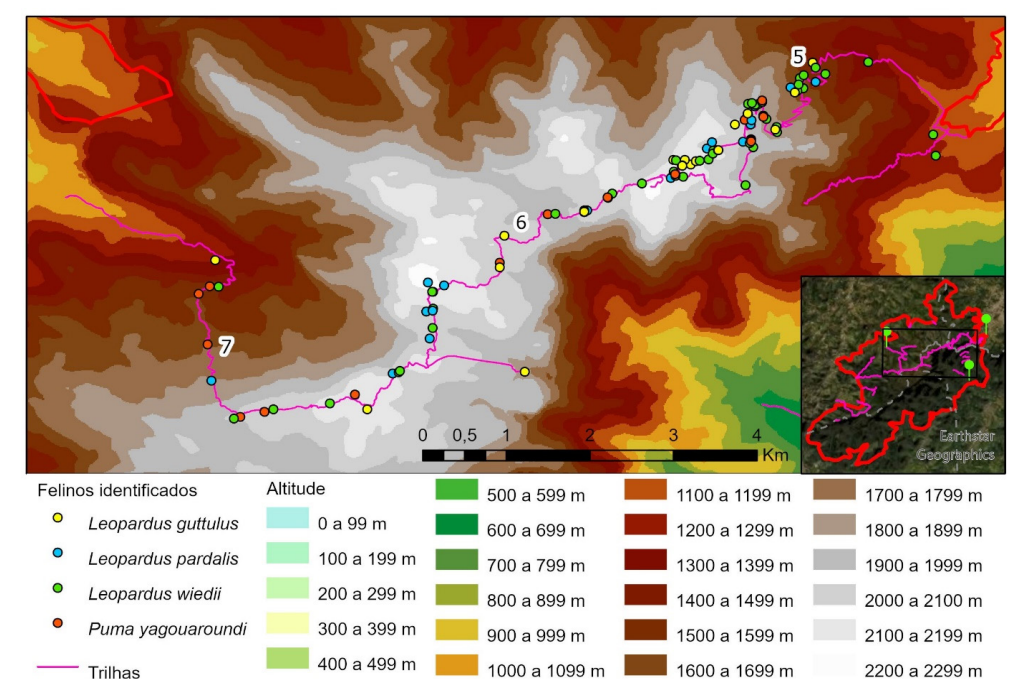

Figura 4 - Distribuição das amostras fecais identificadas atribuídas a felinos em relação à altitude, com destaque para as trilhas com mais de 10 ocasiões de coleta: 5) Trilha da Pedra do Sino; 6) Trilha Açu-Sino; e 7) Trilha para o Açu.

Considerando-se apenas as trilhas com mais de 10 ocasiões amostrais, a distribuição de amostras/ocasião encontradas em cada faixa altitudinal não diferiu significativamente do esperado, dado o perfil altimétrico destas trilhas $(\mathrm{X} 2=15.315$, g.l. $=12, \mathrm{p}=0.2247$; Fig. 5).

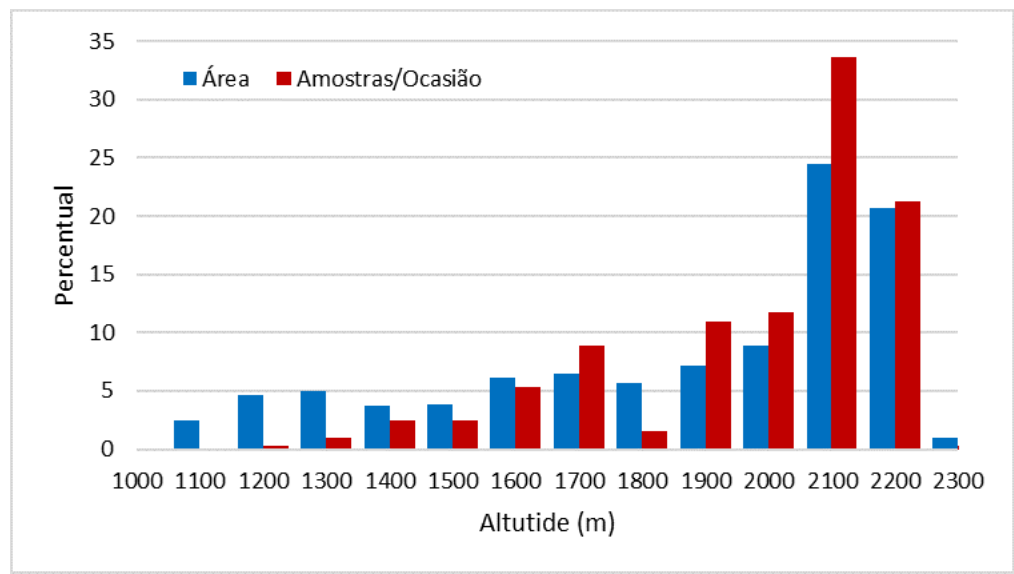

Figura 5 - Distribuição do percentual de área e amostras/ocasião em cada faixa altitudinal entre 1000 e $2.300 \mathrm{~m}$ de altitude, nas trilhas com mais de 10 ocasiões amostrais cada. 


\section{Discussão}

Todas as cinco espécies de felinos com ocorrência atual conhecida para o PARNASO (Cronemberger et al., 2019) foram identificadas nas amostras fecais, sendo que a maior parte foi atribuída a gatos pintados, pertencentes ao gênero Leopardus (85\% das amostras de felinos). Destas, mais da metade foram identificadas como pertencentes a Leopardus wiedii (54\%), mais que a soma das amostras atribuídas às outras duas espécies do gênero, $L$. pardalis $(23 \%)$ e $L$. guttulus (22\%). Entre 2010 e 2017, armadilhas fotográficas fizeram 234 registros de gatos pintados no PARNASO (Nagy-Reis et al., 2020). Entre os 85 registros identificados ao nível de espécie, L. wiedii também aparece com um número de registros bastante superior aos de seus congêneres (78\%), enquanto L. guttulus foi identificado em $18 \%$ e L. pardalis em apenas 5\% dos registros.

Leopardus wiediiédescrito naliteratura como um gato muito associado a ecossistemas florestais, sendo mais adaptado para viver em árvores que as outras espécies de gatos neotropicais, embora em geral se desloque pelo solo (Espinosa et al., 2018; Oliveira et al., 2013, 2015). O limite superior reconhecido de sua distribuição altitudinal é $1500 \mathrm{~m}$, embora haja alguns registros até cerca de $3000 \mathrm{~m}$ de altitude nos Andes (Oliveira et al. 2015). No presente estudo, quase todas as amostras atribuídas a $L$. wiedii foram encontradas acima de $1500 \mathrm{~m}$, de forma recorrente ao longo dos anos, indicando um uso frequente desta região. Embora o gato-maracajá apresente adaptações que facilitem a exploração do estrato arbóreo das florestas, a predominância de vegetação herbáceoarbustiva na porção mais alta do PARNASO não parece inibi-lo de usar com frequência essas áreas.

Apesar de Puma concolor ser o felino mais abundante nos registros feitos por armadilhas fotográficas no PARNASO (Nagy-Reis et al., 2020), a coleta de suas fezes foi rara, sendo o felino menos identificado por meio de tricologia. Puma concolor é a maior espécie de felino na área de estudo, com os maiores territórios, e assim percorre uma área maior que as outras espécies, aumentando a chance de ser registrada por armadilhas fotográficas $e$ diminuindo a chance de que suas fezes sejam encontradas (Tomas et al., 2004).
Uma pequena parte dos pelos analisados foi atribuída a outros mamíferos. Embora sejam poucos, esses registros são muito importantes, pois foram identificadas espécies com poucos registros por armadilhas fotográficas, o outro método de inventário e monitoramento de mamíferos adotado no PARNASO, como o furão (Galictis cuja) e o cateto (Pecari tajacu), ou mesmo sem qualquer registro por este método, como a lontra (Lontra longicaudis) e o mão-pelada (Procyon cancrivorus). Os oito registros de pelo de furão superam numericamente os três registros fotográficos da espécie até 2017 (Nagy-Reis et al., 2020). O único registro de pelo de cateto se deu na trilha do Rancho Frio, uma das áreas onde já havia registro fotográfico, porém dois anos após a última foto, constituindo-se no registro mais recente da espécie nessa área. Antes dos registros por tricologia feitos no presente estudo, a ocorrência de L. longicaudis era conhecida por visualizações esporádicas e de $P$. cancrivorus apenas por pegadas encontradas na década de 1940 (Cunha, 2007; Davis, 1945).

As coletas de amostras se concentraram nas altitudes mais elevadas do PARNASO, marcadamente acima de $2000 \mathrm{~m}$ de altitude, pois as trilhas onde houve mais coletas são também as trilhas com trechos mais elevados. A paisagem dos campos de altitude, formação predominantemente herbáceo-arbustiva com muitos afloramentos rochosos, favoreceu a visualização do material fecal. Na maioria dos casos, nos campos de altitude, as fezes foram encontradas em posição de destaque sobre as pedras. Carnívoros terrestres costumam depositar suas fezes em locais visíveis, como cruzamento de trilhas e estradas vicinais, ou sobre barrancos e outros locais elevados como estratégia de comunicação intra e interespecífica e marcação territorial (Chame, 2003; Harmsen et al., 2010).

Em comparação, o fato de poucas amostras terem sido encontradas nas áreas de florestas úmidas pode estar relacionado à sua menor conspicuidade em solo florestal e ao menor tempo de permanência das fezes nestes ambientes, onde se decompõem mais depressa (Abrams et al., 2018), e não a uma preferência por ambientes abertos, a julgar pelo grande número de registros das mesmas espécies em armadilhas fotográficas em áreas florestais no PARNASO (Aximoff et al., 2015; Nagy-Reis et al., 2020). A adição ao protocolo de 
campo do registro da distância da amostra para o leito da trilha pode ajudar a esclarecer o papel da paisagem na detectabilidade das fezes, além de permitir a análise dos resultados pelo método de amostragem de distância, o que permitiria gerar informações sobre abundância das espécies de felinos (Tomas et al., 2004).

O sucesso do registro de fezes de felinos nos campos de altitude contrasta com a pequena quantidade de registros por armadilhas fotográficas na mesma área (Nagy-Reis et al., 2020). Por ser uma fisionomia aberta, com predomínio de vegetação herbáceo-arbustiva e muitos afloramentos rochosos, o método de armadilhamento fotográfico apresenta limitações em comparação com ambientes florestais, como maior dificuldade de prever rotas de deslocamento de animais e de fixar as armadilhas em troncos, bem como maior proporção de disparos em falso devido ao vento. Essas características podem explicar as diferenças na detectabilidade de felinos pelos dois diferentes métodos nos campos de altitude, onde a tricologia permitiu o registro de quatro das cinco espécies de felinos em localidades sem registro anterior por armadilhas fotográficas. $H$. yagourandi foi registrado em duas localidades exclusivamente por este método, enquanto $L$. pardalis, $L$. wiedii e L. guttulus em uma localidade cada.

O fato de fezes de quatro diferentes espécies de felinos (Leopardus wiedii, L. guttulus, L. pardalis e Herpailurus yagouaroundi) terem sido encontradas com frequência nas mesmas áreas, principalmente nas trilhas do Açu, Açu-Sino e da Pedra do Sino, parece indicar que ocorre sobreposição de área de vida entre estas espécies. Essas espécies são sabidamente simpátricas, porém a maneira como dividem seus nichos, permitindo sua coexistência, ainda não é completamente compreendida (Sanchez-Barradas \& Villalobos, 2020; Sunquist \& Sunquist, 2002). Em escala local, são considerados fatores importantes o padrão temporal de atividade e a dieta (Bianchi et al., 2016; Di Bitetti et al., 2010; Wang, 2002). A análise do conteúdo das amostras fecais pode ajudar a entender o papel da sobreposição de dieta na partição dos nichos, enquanto estudos com armadilhas fotográficas provém dados para avaliar as diferenças no padrão de atividades.

Embora tenham sido coletadas amostras de fezes com características morfológicas compatíveis com felinos (cilíndricas, afiladas em uma das extremidades), que costumam conter muitos pelos
(Chame, 2003), não foi possível recuperar pelosguarda em $39 \%$ das amostras analisadas, apesar de haver sempre muitos pelos nelas. Em alguns casos, essas estruturas encontravam-se deterioradas, não sendo possível imprimir a cutícula ou diafanizar de forma adequada a medula. Essa é uma limitação do método, encontrada em outros estudos que analisaram amostras fecais coletadas em campo na Mata Atlântica usando a tricologia. RochaMendes e colaboradores (2010) e Santos (2014) obtiveram sucesso na identificação de pelosguarda de mamíferos em $47 \%$ das amostras que analisaram, uma taxa menor que no presente estudo; enquanto Bianchi e colaboradores (2011) obtiveram sucesso similar (66\%), e Wang (2002) obteve sucesso maior (93\%). Essa diferença pode ser explicada, pelo menos em parte, pela qualidade do material fecal coletado, que pode ficar comprometida com o tempo de permanência em campo antes da coleta, devido a fatores como a lixiviação pela chuva e exposição solar, muito intensa nos campos de altitude (Dib et al. 2019). Apesar disso, é importante coletar amostras em todas as estações para avaliar mudanças no uso do espaço e na dieta ao longo do tempo. É importante destacar também que os parques são locais abertos à visitação e que os turistas podem pisotear o bolo fecal nas trilhas, comprometendo a sua morfologia e acelerando sua decomposição.

A combinação de diferentes técnicas de identificação das amostras fecais, como morfologia do material fecal, tricologia dos pelos-guarda $e$ análises moleculares do material genético extraído do sedimento fecal, aumenta o percentual de amostras coletadas identificadas, além de aumentar a confiabilidade dos resultados (Alberts et al., 2017; Dib et al., 2020; Farrell et al., 2000). A nova etapa do projeto, já em andamento, prevê a adição de técnicas moleculares na identificação as amostras. Resultados preliminares mostraram que é possível isolar DNA das amostras de fezes coletadas $e$ armazenadas de acordo com os protocolos descritos neste trabalho e permitiram selecionar um protocolo ideal para a continuidade do projeto (Loh, dados não publicados).

A coleta oportunística de amostras fecais em campo é uma atividade simples, que pode ser realizada ao se percorrer as trilhas com objetivos diversos (Alberts et al., 2017), e que pode trazer resultados interessantes para complementar inventários, monitoramento e outras pesquisas. Com um treinamento simples e materiais baratos, 
funcionários de unidades de conservação podem realizar a coleta em conjunto com suas atividades rotineiras, aumentando a probabilidade de encontrar amostras em relação a coleta feita por pesquisadores, que estão na UC em poucas ocasiões.

No PARNASO, a coleta de amostras começou como atividade esporádica em 2009 e passou a ser uma atividade sistemática em 2011, quando o primeiro aluno do Programa de Iniciação Científica (PIBIC) começou suas atividades. A quantidade de amostras coletadas $e$ analisadas foi maior nos anos em que havia a participação de alunos PIBIC no projeto, tanto porque os próprios alunos percorreram as trilhas em busca de amostras quanto porque motivaram os demais funcionários a procurar amostras em suas atividades cotidianas. Nos anos em que não houve alunos de iniciação científica, a coleta de amostras diminuiu, apesar de a equipe do parque ter percorrido as mesmas trilhas rotineiramente. Fica evidente a necessidade de manter a equipe treinada e motivada para garantir a continuidade do projeto, uma vez que amostras podem ser armazenadas por longos períodos antes de serem processadas, $e$ podem ser utilizadas para múltiplos propósitos.

Além da presença e uso do espaço, outras informações importantes para a conservação dos felinos podem ser obtidas a partir de suas amostras fecais, como sua dieta (Bianchi et al., 2011; Rocha-Mendes et al., 2010; Santos, 2014; Wang, 2002), saúde (Dib et al., 2018, 2020), razão sexual e estimativas de tamanho populacional (Miotto et al., 2014; Pilgrim et al., 2005), bem como a avaliação de parentesco entre indivíduos e o grau de isolamento populacional (Miotto et al., 2011; Saranholi et al., 2017). Além disso, pelos de mamíferos são bons acumuladores de substâncias contaminantes devido ao seu crescimento lento, $e$ carnívoros, como predadores de topo de cadeia, estão entre os organismos mais afetados pela magnificação trófica de poluentes, como mercúrio e poluentes orgânicos persistentes (Carvalho et al., 2020; Lopes et al., 2020).

Um estudo recente com roedores $e$ marsupiais, presas comuns a muitas espécies de felinos, coletados nos campos de altitude do PARNASO, justamente onde se concentram as amostras fecais encontradas no presente estudo, indicou a presença de diferentes concentrações de mercúrio no tecido hepático destes animais, sugerindo a ocorrência de transferência trófica do mercúrio (Lucena, 2018). No entanto, ainda é necessário avaliar se a quantidade de pelos recuperados nas amostras fecais é suficiente para as análises de estudos de bioacumulação. Para responder a algumas destas perguntas, a coleta oportunística de amostras fecais pode não ser suficiente, sendo necessário estabelecer um protocolo de amostragem focado na busca por fezes, com registro do esforço amostral; isto, porém, não reduz a relevância de coletar $e$ armazenar amostrais fecais de maneira oportunística.

\section{Conclusão}

A identificação de mamíferos por meio de análise microscópica de pelos-guarda recuperados de amostras fecais trouxe novas informações sobre a mastofauna do PARNASO, como novas localidades, a frequente presença de Leopardus wiedii acima de $1500 \mathrm{~m}$ de altitude, $e$ registros de espécies pouco documentadas na UC, como Procyon cancrivorous. $\mathrm{O}$ sucesso de coleta de fezes na porção mais alta do parque, os campos de altitude, reflete o esforço empregado, porém traz a possibilidade de complementar outros métodos que apresentam menor sucesso neste ambiente.

A coleta de amostras fecais em campo é uma atividade simples e barata, que pode facilmente ser incorporada às atividades cotidianas de funcionários de unidades de conservação. As amostras podem ser armazenadas por longos períodos e analisadas por diversas técnicas por instituições parceiras. Uma mesma amostra pode servir a diversos objetivos de pesquisa e, quanto mais técnicas diferentes forem combinadas, maior será o valor das informações reveladas para o monitoramento da saúde ambiental e a conservação das espécies de carnívoros silvestres, muitos deles ameaçados de extinção. Apesar disso, ainda são escassos os projetos que utilizam esse método como ferramenta de monitoramento em unidades de conservação.

Para UCs que pretendam iniciar um projeto de monitoramento de mamíferos por meio de análises fecais, recomendamos: a) trabalhar em parceria com instituições de pesquisa locais, buscando sempre o melhor aproveitamento possível das amostras; b) registrar o esforço sistemático de busca de amostras, sem, no entanto, descartar o uso de amostras coletadas oportunisticamente; c) incluir no protocolo de campo o registro da distância da amostra coletada para a trilha. 


\section{Agradecimentos}

Este artigo foi elaborado por vários grupos de pesquisa que atuaram de forma conjunta com a equipe do PARNASO de 2009 a 2019. Agradecemos a Fabiane Pereira e a Jorge Luiz do Nascimento, pelo apoio incondicional à pesquisa no PARNASO. A todos os coletores de amostras fecais no PARNASO. Ao ICMBio, pelas autorizações SISBIO 24613 e 38070, pelas bolsas concedidas pelo Programa de Iniciação Científica (PIBIC/ ICMBio) a R.C.M e M.L.D e pelo financiamento (DIBIO/ICMBio Projeto PEM 011.034). H.G.B. agradece as bolsas e financiamentos do $\mathrm{CNPq}$, FAPERJ e Prociência/UERJ. A CEUA - Fiocruz pela licença LW 53/13 (P-24/13.7). Ao Instituto Federal de Educação Ciência e Tecnologia do Rio de Janeiro (IFRJ), pelo financiamento no âmbito do programa PROCIÊNCIA. CC agradece a ESRI pelo Conservation Grant. Aos revisores anônimos pelas valiosas sugestões.

\section{Referências}

Abrams JF et al. Studying terrestrial mammals in tropical rainforests. A user guide for camera-trapping and environmental DNA. Leibniz-IZW, Berlin, Germany. 2018.

AFC DAAC, 2014. Imagem ALOS PALSAR, L-Band, Cena ALPSRP269566730. Includes Material (C) JAXA/METI 2008. DOI: https://doi.org/10.5067/ Z97HFCNKR6VA

Ahumada JA et al. Community structure and diversity of tropical forest mammals: Data from a global camera trap network. Philosophical Transactions of the Royal Society B: Biological Sciences, 366(1578): 2703-2711, 2011.

Alberts CC, Saranholi BH, Frei F \& Galetti PM. Comparing hair-morphology and molecular methods to identify fecal samples from Neotropical felids. PLoS One, 12: 1-24, 2017.

Aximoff I, Cronemberger C \& Pereira FA. Amostragem de longa duração por armadilhas fotográficas dos mamíferos terrestres em dois parques nacionais no estado do Rio de Janeiro. Oecologia Australis, 19(1): 215-231, 2015.

Bianchi RC, Rosa AF, Gatti Av \& Mendes SL. Diet of margay, Leopardus wiedii, and jaguarundi, Puma yagouaroundi, (Carnivora: Felidae) in Atlantic Rainforest, Brazil. Zoologia, 28(1): 127-132, 2011

Bianchi RC, Olifiers N, Gomper ME \& Mourão G. Niche partitioning among mesocarnivores in a Brazilian wetland. PLoS ONE 11(9): e0162893. doi:10.1371/ journal.pone.0162893, 2016.
Bruner AG et al. Effectiveness of Parks in Protecting Tropical Biodiversity. Science, 125(5501): 125-128, 2001.

Carvalho GO et al. Road-killed giant anteater's fur as a tool for biomonitoring mercury contamination. Chemosphere. DOI: 10.1016/j. chemosphere.2020.128644. 2020.

Chame M. Terrestrial Mammal Feces: A Morphometric Summary and Description. Memórias do Instituto Oswaldo Cruz, 98: 71-94, 2003.

Cronemberger $C$ et al. 2019. Mamíferos do Parque Nacional da Serra dos Órgãos: Atualização da lista de espécies e implicações para a conservação. Oecologia Australis, 23: 191-214, 2019.

Cronemberger C \& Viveiros de Castro EB. Ciência e Conservação na Serra dos Órgãos. Brasília: IBAMA. 296p. 2007.

Cullen Jr L, Abreu CK, Sana D \& Nava AFD. As onçaspintadas como detetives da paisagem no corredor do Alto Paraná, Brasil Natureza e Conservação 3: 43-58, 2005.

Cunha AA. Alterações na composição da comunidade e o status de conservação dos mamíferos de médio e grande porte da Serra dos Órgãos. In: Cronemberger $\mathrm{C}$, Viveiros de Castro EB. Ciência e Conservação na Serra dos Órgãos. Brasília: IBAMA: 211-224, 2007.

Davis DE. The Annual Cycle of Plants, Mosquitoes, Birds, and Mammals in Two Brazilian Forests. Ecological Monographs, 15(3), 243-295, 1945.

Dib LV et al. Gastrointestinal parasites among felids inhabiting the Serra dos Órgãos National Park, Rio de Janeiro, Brazil. Revista Brasileira de Parasitologia, 27(2): 131-140, 2018.

Dib LV et al. Noninvasive Sampling: Monitoring of Wild Carnivores and Their Parasites, p. 1-13. In: Ahmad NB, Mohd NS. (orgs). Protected Areas, National Parks and Sustainable Future. Londres: IntechOpen. 570p. 2019.

Dib LV et al. Noninvasive fecal sampling in Itatiaia National Park, Brazil: wild mammal identification and parasite detection. Dryad. 2: Dataset, 2020.

Di Bitetti MS, De Angelo CD, Di Blanco YE \& Paviolo A. Niche partitioning and species coexistence in a Neotropical felid assemblage. ActaOecologica, 36: 403412, 2010.

Elbroch LM, Quigley HB \& Caragiulo A. Spatial associations in a solitary predator: using genetic tools and GPS technology to assess cougar social organization in the Southern Yellowstone Ecosystem. Acta Ethologica, 18: 127-136, 2015.

Espinosa CC et al. Geographic distribution modeling of the margay (Leopardus wiedii) and jaguarundi (Puma yagouaroundi): a comparative assessment. Journal of Mammalogy, 99(1): 252-262, 2018. 
ESRI. ArcGIS Pro: Release 2.6.3. Redlands, CA: Environmental Systems Research Institute. 2020.

Farrell LE, Roman J \& Sunquist ME. Dietary separation of sympatric carnivores identified by molecular analysis of scats. Molecular Ecology, 9(10): 1583-1590, 2000.

Fontes BL et al. The local extinction of one of the greatest terrestrial ecosystem engineers, the giant armadillo (Priodontes maximus), in one of its last refuges in the Atlantic Forest, will be felt by a large vertebrate community. Global Ecology and Conservation, 24, e01357, 2020.

Galindo-Leal C, Câmara IG \& Reis E. Mata Atlântica: Biodiversidade, ameaças e perspectivas. São Paulo: Fundação SOS Mata Atlântica. Belo Horizonte: Conservação Internacional. 472 p. 2005.

Galleti M et al. Atlantic Rainforest's Jaguars in Decline. Science, 342(6161): 930, 2013.

Haag $\mathrm{T}$ et al. The effect of habitat fragmentation on the genetic structure of a top predator: loss of diversity and high differentiation among remnant populations of Atlantic Forest jaguars (Panthera onca). Molecular Ecology, 19: 4906-4921, 2010.

Harmsen BJ, Foster RJ, Silver S, Ostro L \& Doncaster CP. Differential use of trails by forest mammals and the implications for camera-trap studies: A case study from Belize. Biotropica, 42: 126-133, 2010.

Jorge MLSP, Galetti PM, Ribeiro MC \& Ferraz KMPMB. Mammal defaunation as surrogate of trophic cascades in a biodiversity hotspot. Biological Conservation, 163: 49-57, 2013

Lindenmayer DB \& Likens GE. Adaptive monitoring: a new paradigm for long-term research and monitoring. Trends in Ecology and Evolution, 24(9): 482-486, 2009.

Lopes MCB et al. Total mercury in wild felids occurring in protected areas in the central Brazilian Amazon. Acta Amazonica, 50: 142-148, 2020.

Lovett GM et al. Who needs environmental monitoring? In a nutshell. The Ecological Society of America, 5(5): 253-260, 2007.

Lucena FM. Pequenos mamíferos como biomonitores da contaminação por mercúrio em duas unidades de conservação montanas (SE-Brasil). Trabalho de conclusão de curso (graduação). Universidade Federal do Rio de Janeiro. 54 p. 2018.

McDonald RI \& Boucher TM. Global development and the future of the protected area strategy. Biological Conservation, 144(1): 383-392, 2011.
Miranda GHB, Rodrigues FHG \& Paglia AP. Guia de Identificação de Pelos de Mamíferos Brasileiros. Brasília: Ciências Forenses. 112 p. 2014.

Miotto R A et al. Genetic diversity and population structure of pumas (Puma concolor) in southeastern Brazil: Implications for conservation in a humandominated landscape. Conservation Genetics, 12(6): 1447-1455, 2011.

Miotto RA, Cervini M, Kajin M, Begotti RA \& Galetti PM. Estimating puma Puma concolor population size in a human-disturbed landscape in Brazil, using DNA mark-recapture data. Fauna e Flora International, 48(2): 250-257, 2014.

Morrison JC et al. Persistence of large mammal faunas as indicators of global human impacts. Journal of Mammalogy, 88(6): 1363-1380, 2011.

Moura RC \&, Faria CC. Protocolo de coleta de amostras fecais de mamíferos terrestres da Ordem Carnivora para estudos de dieta. Boletim da Sociedade Brasileira de Mastozoologia, 64: 22-24, 2012a.

Moura RC \& Faria CC. Coleção de Referência de pelos de Mamíferos do PARNA Serra dos Órgãos. In: Anais do Simpósio Latinoamericano de Coleções Biológicas e Biodiversidade: Conhecimento e Gestão. 97 p. 2012 b.

Nagy-Reis M. et al. Neotropical Carnivores: a dataset of occurrence of carnivores in the Neotropics. Ecology 101 (11), e03128. 2020.

Oliveira TG, Almeida LB, Tortato MA \& Beisiegel BM. Avaliação do risco de extinção do gatomaracajá Leopardus wiedii (Schinz, 1821) no Brasil. Biodiversidade Brasileira, 31(1), 76-83, 2013.

Oliveira TG et al. Leopardus wiedii. The IUCN Red List of Threatened Species 2015: e.T11511A50654216. 2015

Pilgrim KL, MCkelvey KS, Riddle AE \& Schwartz MK. Felid sex identication based on noninvasive genetic samples. Molecular Ecology Notes, 5: 60-61, 2005.

Quadros J. 2002. Identificação microscópica de pêlos de mamíferos brasileiros e sua aplicação no estudo da dieta de carnívoros. Tese (Doutorado em Ciências Zoologia). Universidade Federal do Paraná. 127 p.

Quadros J \& Monteiro-filho ELA. Coleta e preparação de pelos de mamíferos para identificação em microscopia óptica. Revista Brasileira de Zoologia. 23: 274-278, 2006a.

Quadros J \& Monteiro-Filho ELA. Revisão conceitual, padrões microestruturais e proposta nomenclatória para os pêlos-guarda de mamíferos brasileiros. Revista Brasileira de Zoologia, 23: 279-292, 2006 b. 
Rocha-Mendes F, Mikich SB, Quadros J \& Pedro WA. Feeding ecology of carnivores (Mammalia, Carnivora) in Atlantic Forest remnants, Southern Brazil. Biota Neotropical. 10: 21-30, 2010.

Rodríguez-Castro KG, Saranholi BH, Bataglia L, Blanck DV \& Galetti Jr, PM. Molecular species identification of scat samples of South American felids and canids. Conservation Genetics Resources, 1-6, 2020.

RStudio Team. RStudio: Integrated Development for R. RStudio, PBC, Boston, MA <http://www.rstudio. com/>, 2020.

Sanchez-Barradas A \& Villalobos F. Species geographical co-occurrence and the effect of Grinnellian and Eltonian niche partitioning: The case of a Neotropical felid assemblage. Ecological Research, 2020: 1-12, 2020.

Santos J, Paschoal A, Massara R \& Chiarello A. High consumption of primates by pumas and ocelots in a remnant of the Brazilian Atlantic Forest. Brazilian Journal of Biology. 74: 632-641, 2014.
Saranholi BH, Chávez-Congrains K \& Galetti PM. Evidence of Recent Fine-Scale Population Structuring in South American Puma concolor. Diversity, 9: 1-11, 2017.

Sunquist M \& Sunquist F. Wild Cats Of The World. University of Chicago Press, Chicago. 462 p. 2002.

Tomas WM, Rodrigues FHG \& Fusco R. Técnicas de levantamento e monitoração de populações de carnívoros. Corumbá: Embrapa Pantanal, 34 p. 2004.

Wang E. Diets of Ocelots (Leopardus pardalis), Margays (L. wiedii), and Oncillas (L. tigrinus) in the Atlantic Rainforest in Southeast Brazil, Studies on Neotropical Fauna and Environment, 37(3): 207-212, 2002.

Wearn OR \& Glover-Kapfer P. Camera-trapping for conservation: a guide to best-practices. WWF Conservation Technology Series. 1: 1-180, 2017.
Biodiversidade Brasileira - BioBrasil.
Edição Temática: PIBIC

n. 1, 2022

http://www.icmbio.gov.br/revistaeletronica/index.php/BioBR

Biodiversidade Brasileira é uma publicação eletrônica científica do Instituto Chico Mendes de

Conservação da Biodiversidade (ICMBio) que tem como objetivo fomentar a discussão e a disseminação de experiências em conservação e manejo, com foco em unidades de conservação e espécies ameaçadas.

ISSN: 2236-2886 